\title{
Role of carbon nanoparticle suspension in sentinel lymph node biopsy for early-stage cervical cancer: a prospective study
}

\author{
Ya Xie ${ }^{1}$, Qian Wang ${ }^{1}$, Huiqing Li $^{1}$, Haixiao Wen ${ }^{1}$, Weiwei Zhang ${ }^{1}$, Jing Bai ${ }^{1}$, Lei $\mathrm{Cai}^{1}$, \\ Jianping Yang ${ }^{1}$, Shuping Yan $^{1}$, Jiaya $\mathrm{Mo}^{1}$, Dong Wang ${ }^{1}$, and Ruixia Guo ${ }^{1}$ \\ ${ }^{1}$ Zhengzhou University First Affiliated Hospital
}

May 5, 2020

\begin{abstract}
Abstract Objective To evaluate the clinical diagnostic validity of carbon nanoparticle suspension (CNS) in sentinel lymph node biopsy (SLNB) for assessing lymphatic spread of early-stage cervical cancer. Design A prospective study. Setting and population 356 cases. Methods We enrolled 356 stage Ia2-IIa2 cervical cancer patients to undergo SLNB using CNS followed by systematic pelvic lymphadenectomy. All lymph node specimens were assessed using conventional histopathologic \pm pathologic ultrastaging analyses. Main outcome measures SLN detection rate (DR), clinical diagnostic validity, and various related factors were analysed. Results CNS identified 1456 SLNs in 325 patients. The overall SLN DR was 91.29\%. A significantly higher DR was found for patients with tumours $<20 \mathrm{~mm}(97.75 \%$ vs. $71.91 \%$; $=0.000)$. Two patients had false-negative results, accounting for $0.615 \%$ of patients who had successful SLN detection. SLNB with CNS had sensitivity of $92.86 \%$, false-negative rate (FNR) of $7.14 \%$, and negative predictive value (NPV) of $99.29 \%$. Importantly, sensitivity (100\%), NPV (100\%), and FNR (0\%) were improved when testing the subgroup of patients with tumours $<20 \mathrm{~mm}$ (267 cases). There were no observed differences in DR based on pathologic type or grade, stage, depth of stromal invasion, surgical approach, menopausal status, or prior treatment with chemotherapy $(\mathrm{p}>0.05)$. Conclusions SLNB with CNS results in favourable DR, sensitivity, and NPV for women with early-stage cervical cancer with small tumour sizes. SLNB with CNS is safe, feasible, and relatively effective for guiding precise surgical treatment of early-stage cervical cancer. Keywords SLNB, CNS, early-stage cervical cancer
\end{abstract}

Role of carbon nanoparticle suspension in sentinel lymph node biopsy for early-stage cervical cancer: a prospective study

\section{Running title: Role of CNS in SLNB for early-stage cervical cancer}

Xie Ya $^{a^{*}}$ Wang Qian $^{a}$ Li Huiqing $^{a}$ Wen Haixiao $^{a}$ Zhang Weiwei $^{a}$ Bai Jing ${ }^{a}$ Cai Lei ${ }^{a}$ Yang Jianping $^{b}$ Yan Shuping ${ }^{b}$ Mo Jiaya ${ }^{a}$ Wang Dong ${ }^{a}$ Guo Ruixia ${ }^{a \#}$

${ }^{a}$ Department ofObstetrics and Gynecology, The First Affiliated Hospital of Zhengzhou University, Zhengzhou 450o52, China

${ }^{b}$ Department of Pathology, The First Affiliated Hospital of Zhengzhou University, Zhengzhou 45oo52, China

${ }^{*}$ Dr. Xie Ya is the first author.

\# Corresponding author: Prof. Guo Ruixia

Postal address: No.1, Eastern Jianshe Road, Erqi District, Zhengzhou City, Henan Province, China 450052.

Telephone number: 0086-13525569376

Fax number: 0086-0371-66970906

Email address:fccguorx@zzu.edu.cn 


\section{Abstract \\ Objective}

To evaluate the clinical diagnostic validity of carbon nanoparticle suspension (CNS) in sentinel lymph node biopsy (SLNB) for assessing lymphatic spread of early-stage cervical cancer.

Design

A prospective study.

\section{Setting and population}

356 cases.

\section{Methods}

We enrolled 356 stage Ia2-IIa2 cervical cancer patients to undergo SLNB using CNS followed by systematic pelvic lymphadenectomy. All lymph node specimens were assessed using conventional histopathologic \pm pathologic ultrastaging analyses.

\section{Main outcome measures}

SLN detection rate (DR), clinical diagnostic validity, and various related factors were analysed.

\section{Results}

CNS identified 1456 SLNs in 325 patients. The overall SLN DR was 91.29\%. A significantly higher DR was found for patients with tumours $<20 \mathrm{~mm}(97.75 \%$ vs. $71.91 \%$; $\mathrm{p}=0.000)$. Two patients had false-negative results, accounting for $0.615 \%$ of patients who had successful SLN detection. SLNB with CNS had sensitivity of $92.86 \%$, false-negative rate (FNR) of $7.14 \%$, and negative predictive value (NPV) of $99.29 \%$. Importantly, sensitivity (100\%), NPV (100\%), and FNR (0\%) were improved when testing the subgroup of patients with tumours $<20 \mathrm{~mm}$ (267 cases). There were no observed differences in DR based on pathologic type or grade, stage, depth of stromal invasion, surgical approach, menopausal status, or prior treatment with chemotherapy $(\mathrm{p}>0.05)$.

\section{Conclusions}

SLNB with CNS results in favourable DR, sensitivity, and NPV for women with early-stage cervical cancer with small tumour sizes. SLNB with CNS is safe, feasible, and relatively effective for guiding precise surgical treatment of early-stage cervical cancer.

Keywords SLNB, CNS, early-stage cervical cancer

\section{Tweetable abstract}

Sentinel lymph node biopsy with carbon nanoparticle suspension is safe and feasible for early-stage cervical cancer.

\section{Introduction}

Cervical cancer is the second most commonly diagnosed cancer and the third leading cause of cancer-related deaths among females, with significant upward trends in its incidence and mortality ${ }^{1-2}$. Conventionally, women with early-stage cervical cancer (FIGO 2009 stages Ia2-IIa2) undergo hysterectomy with pelvic lymph node dissection (PLND). However, early-stage cervical cancer with nodal metastases accounts for only 8 $26 \%$ of cases $^{3}$, suggesting that approximately more than $75 \%$ of those patients routinely undergo unnecessary PLND. PLND increases the incidence of short- and long-term complications, especially leg lymphedema, and removing "healthy" lymph nodes may negatively influence the immune system ${ }^{4}$. All complications markedly decrease the quality of post-operative life. Hence, avoiding unnecessary PLND in early-stage cervical cancer patients is clinically important. 
The so-called sentinel lymph node (SLN) is the first lymph node to drain from a primary tumour ${ }^{5}$. If SLNs are metastasis-negative, then upstream nodes should (in theory) also be metastasis-free. To reduce the morbidity associated with PLND, SLN biopsy (SLNB) is diagnostically beneficial when assessing for nodal metastases in early-stage cervical cancer ${ }^{6}$. The National Comprehensive Cancer Network (NCCN) guidelines recommend SLNB as an alternative to PLND (Recommendation 2A) ${ }^{7}$. However, the implementation of SLNB is limited, especially in China, where one-fourth of the cervical cancer cases in the world occur ${ }^{1}$. According to an anonymous online survey completed by all full and candidate members of the Society of Gynaecology and Obstetrics (SGO), only 70\% ( $\mathrm{n}=139)$ of the 198 respondents (response rate $=17.7 \%$ ) indicated that they perform SLNB. The main reason for not performing SLNB was the lack of standardized training and technology ${ }^{8}$. Inevitable heterogeneity was observed in patient selection and tracer application in previous studies, which were mainly retrospective studies with small sample sizes. Moreover, there are some disadvantages to using the most common tracers, such as allergic reactions, radioactive pollution, and the cost of and dependence on expensive imaging technology ${ }^{9-11}$.

Therefore, we investigated carbon nanoparticle suspension (CNS), a new tracer that can be used in SLNB for patients with early-stage cervical cancer. A previous study showed that CNS permits easy identification, has good lymphatic tracer effects, and results in increased lymph node retrieval ${ }^{12}$. Its use in SLNB for cervical cancer is relatively new. Accordingly, the objectives of this study were to assess the safety and feasibility of CNS in SLNB, and to determine the diagnostic validity of the SLN status for assessing lymphatic spread in early-stage cervical cancer.

\section{Methods}

\section{Selection of patients}

Patients with early-stage cervical cancer were eligible for this prospective study. A total of 356 patients were consecutively enrolled. They underwent SLNB followed by standard treatment, according to institutional guidelines (modified radical hysterectomy/radical hysterectomy plus complete pelvic lymphadenectomy, with or without para-aortic lymph node sampling), during robotic or laparoscopic-assisted surgery between May 2017 and December 2019. The inclusion criteria were as follows: FIGO 2009 stages Ia2-IIa2; squamous cell carcinoma or typical adenocarcinoma (HPV-related); no evidence of bulky or suspicious PLNs, or distant metastases according to conventional imaging studies; surgery as the primary treatment; and the provision of signed informed consent. The exclusion criteria were as follows: age younger than 18 years or older than 70 years; pregnancy; incidence of other tumours within 5 years; contra-indication for surgical treatment; and intra-operative evidence of more advanced disease (>IIa2). Given the learning curve of SLNB, the first 30 patients enrolled were not included in the final statistical analysis ${ }^{13}$.

\section{Tracer administration}

Before injection, the retroperitoneum was opened and lymph node basins were viewed. Withdrawal was first performed to prevent injection into blood vessels, and SLNB was performed by injecting CNS (China Food and Drug Administration approval H20073246; Lai Mei Pharmaceutical Co., Chongqing, China) into the vaginal portion of the cervix at the 3 and 9 o'clock positions. The injection was administered superficially by a skin test needle approximately $5-10 \mathrm{~mm}$ from the tumour border and perpendicular to the cervical surface. According to our experience, to achieve better staining, the total volume of the CNS was $0.5 \mathrm{~mL}$, with approximately $0.25 \mathrm{~mL}$ administered at each point. The needle was inserted approximately $2-3 \mathrm{~mm}$, and the suspension was slowly injected over the course of approximately $3 \mathrm{~min}$ (Figure 1.A). To prevent leakage, the syringe was kept in place for a few seconds after the application. Then, lymphatic drainage of the CNS was observed. The black-stained lymphatic duct resulted in black-stained lymph nodes. SLN was defined as the first lymph node(s) or group of lymph nodes that the CNS drained into, as recognised by black staining through direct visualisation within 15 minutes after completing the injection (Figure 1.B). The time required for SLN mapping was calculated from the time of injection completion to black-staining of the SLN. Subsequently, SLNs were located and removed separately from each side of the pelvis. Hysterectomy with complete pelvic lymphadenectomy was also performed. Adverse reactions were recorded during and after the 
injection of the tracer. All surgeries were performed by the same experienced surgeons.

\section{Histopathological evaluation}

After surgery, all SLNs and other surgical specimens were fixed in $10 \%$ neutral-buffered formalin, cut into 2 -mm blocks, and embedded in paraffin. Sections $(4 \mu \mathrm{m})$ obtained from each block of lymph nodes were then submitted for a routine pathological assessment after haematoxylin and eosin staining (HE), examined by pathologists specialising in gynaecological oncology. False-negative cases were defined as the hemi-pelvis being negative for SLNs but positive for non-SLNs in the same pelvic side. Since the goal of our study was not to provide deeper insight into the problems associated with pathologic ultrastaging on low-volume metastasis, if an SLN had metastasis in the initial HE-stained section, no further work-up was performed on that lymph node. If SLN was negative in the initial section, but with metastasis in non-SLN, then the ideal ultrastaging protocol was carried out on the whole SLN, which included consecutive sections $(4 \mu \mathrm{m})$ obtained in regular 150- $\mu \mathrm{m}$ intervals, and immunohistochemical staining using antibodies against cytokeratins (AE1/AE3, 1:50 dilution; Dako, Glostrup, Denmark) to identify micrometastasis ${ }^{14}$. For other cases with SLNs that were metastasis-free in the initial HE, an additional simple ultrastaging protocol was performed to examine the SLN for low volume metastatic disease: two consecutive sections (4- $\mu$ m thick) in regular $150-\mu \mathrm{m}$ intervals and six additional sections from each block were stained with HE and immunohistochemical staining (Figure $2)^{15}$.

\section{Statistical analysis}

All statistical analyses were performed using SPSS software (version 21.0; IBM for Windows, Armonk, NY, USA). Categorical variables were analysed using the chi-square test (or Continuity correction chi-square test or Fisher's exact test). The stepwise multivariate Logistic model using the forward Wald method was used to determine independent impact factors for lymph node metastasis. $<0.05$ was considered statistically significant.

\section{Results}

\section{Patient characteristics and complications of CNS}

A total of 356 patients with early-stage cervical cancer underwent SLNB with CNS injection, followed by standard treatment. The time required for SLN mapping was $8.7 \pm 1.5 \mathrm{~min}$ (range: $4.5-15.0 \mathrm{~min}$ ). Adverse reactions to CNS were rare, and no patient exhibited an anaphylactic reaction or serious adverse events related to CNS. A low-grade fever occasionally occurred following injection intra-operatively $(1.12 \%)$; however, it did not require special treatment. Patient demographics and tumour characteristics are summarised in Table 1. The mean age of the patients was 46 years (range: 23-68 years). Histology primarily indicated squamous carcinoma (89.04\%).

\section{SLNB DR and the analysis of impact factors on the SLNB DR}

Of the 356 patients enrolled, 325 had at least one SLN that was successfully stained black by CNS. The overall SLNB detection rate (DR) was $91.29 \%(325 / 356)$. SLNB was performed in both sides of the pelvis in 252 patients, resulting in a bilateral DR of $70.79 \%$. A total of 8873 lymph nodes and 1456 SLNs were removed from 356 patients. The average numbers of lymph nodes and SLNs removed from each patient were 24.92 and 4.48, respectively (Table S1). The total number of SLNs accounted for $16.41 \%$ of all lymph nodes (1456/8873). A dramatically higher overall DR was observed for those with smaller tumour sizes $(<20 \mathrm{~mm})$ $(97.75 \%$ vs. $71.91 \% ; \mathrm{p}=0.000)$, and for those without lympho-vascular space invasion (LVSI) $(94.07 \%$ vs. $85.83 \% ; \mathrm{p}=0.009)$. However, no differences based on age, histologic type or grade, stage, depth of stromal invasion, surgical approach, menopausal status, or prior chemotherapy treatment were observed in the overall SLN DR ( $\mathrm{p}>0.05)$. A relatively higher SLN DR was found for patients with stage Ia2-Ib1 disease $(93.05 \%$ vs. $86.60 \% ; \mathrm{p}=0.055)$, squamous carcinoma ( $92.43 \%$ vs. $82.05 \%$; $=0.062)$, well-differentiated tumours $(98.55 \%$ vs. $90 \%$ and $88.66 \% ; \mathrm{p}=0.055)$, and without deep stromal invasion (DSI) $(93.58 \%$ vs. $88.76 \%$; $=0.107)$ (Table 1). 


\section{Locations of SLNs in the pelvis}

The locations of all SLNs identified were in both sides of the pelvis (Figure1.C). The majority of SLNs $(85.71 \%)$ were found in the pelvis along the iliac vessels and in the obturator space: external iliac, 486 (33.38\%); obturator, 438 (30.08\%); the common iliac, 217 (14.90\%); and the internal iliac, 107 (7.35\%). Furthermore, we found SLNs in some unusual anatomic sites, including the presacral (4.33\%) and parametrial $(3.02 \%)$ regions (Table S2).

\section{Diagnostic value of SLNB}

Among the 325 patients, $46(12.13 \%)$ had 133 metastatic lymph nodes. A total of 44 patients had SLN metastases, thereby accounting for $95.65 \%$ of metastatic cases. Of the 325 patients in whom SLNs were successfully identified, 26 patients had SLN metastasis and PLN metastasis, 18 patients had SLN metastasis but no PLN metastasis (including two patients with micro-metastasis in SLNs), two patients had only PLN metastasis and no SLN metastasis, and 279 patients had no metastasis in the SLNs and PLNs. As a result, the sensitivity of SLNB when used to identify nodal metastatic disease was $92.86 \%$. Among the 281 patients with negative SLN results, 279 had true-negative non-SLNs, which resulted in a negative predictive value (NPV) of $99.29 \%$. Two patients had negative SLNs but positive non-SLNs, yielding a false-negative rate (FNR) of $7.14 \%$ (Table 2). The common characteristics of the two false-negative cases were tumour diameter larger than $20 \mathrm{~mm}$ and the presence of DSI and LVSI (Table S3, Figure 1D). We analysed the subgroup of patients with tumours smaller than $20 \mathrm{~mm}$ (267 cases) and obtained an ideal FNR of $0 \%$ and higher DR (97.75\%), sensitivity (100\%), and NPV (100\%). Compared to the [?]20-mm-tumour-size subgroup, the specificity, NPV, and accuracy were markedly improved (Table S4). Furthermore, we found that lymph node metastatic status was significantly associated with tumour size $(\mathrm{p}=0.000)$, depth of stromal invasion $(\mathrm{p}=$ $0.028)$, LVSI $(\mathrm{p}=0.000)$ and stage $(\mathrm{p}=0.000)$, and multivariate analysis showed that tumour size, stage, and LVSI are independent risk factors for lymph node metastasis (Tables S5 and S6).

\section{Discussion}

\section{Main findings}

We obtained promising DR (91.29\%) and high NPV (99.29\%). A higher DR was observed in smaller tumours subgroup $(<20 \mathrm{~mm})$, as well as higher sensitivity $(100 \%)$, higher NPV $(100 \%)$, and no false-negative results.

\section{Strengths and Limitations}

We first demonstrated that SLNB with CNS had appropriate diagnostic validity in a prospective, singlecentre study with a large sample size of 356 patients. The findings suggest that SLNB with CNS is a simple and effective procedure in early-stage cervical cancer, especially for patients with tumours smaller than 20 $\mathrm{mm}$.

Our study has several limitations. SLNB with CNS for the treatment of cervical cancer is still new; thus, multicentre studies should be performed to further confirm our results. We did not apply ideal ultrastaging for all sentinel lymph nodes because of requirements for the preservation of pathological tissue.

\section{Interpretation}

We found that among patients with metastases, 95.65\% had SLN metastases, suggesting that SLN is a rather sensitive assessment indicator of metastatic lymph nodes. Among methods of assessing the PLN status, SLNB is more suitable than imaging techniques ${ }^{16}$. Moreover, compared with PLND, SLNB reduces complications of $\mathrm{PLND}^{17}$, and no difference was observed in the recurrence-free survival rates of PLND and SLNB by a prospective trial ${ }^{18}$. Therefore, SLNB may be used as an alternative to PLND to prevent unnecessary and harmful interventions.

In this study, we explored the new application value of CNS as a promising SLN tracer for SLNB in early-stage cervical cancer. CNS is an advanced nanotechnology that consists of small molecules with lymphatic tropism. CNS with nanoparticles measuring $150 \mathrm{~nm}$ in diameter can pass readily through lymphatic vessels (diameter: 
120-500 $\mathrm{nm}$ ), but not through capillaries (diameter: 20-50 nm). Then, the CNS is identified, engulfed by macrophages, and brought to lymphatic vessels, such that it accumulates in the lymphatic system; therefore, CNS effortlessly stains lymph nodes. No patient exhibited anaphylactic reactions or serious adverse events in this study; this may be because CNS does not enter the bloodstream. Accordingly, SLNB with CNS should be considered safe. In recent years, CNS has proven to be beneficial as a lymph node tracer that allows lymph nodes to be explored during surgery for breast cancer, thyroid cancer, and gastric cancer ${ }^{19-21}$. For cervical cancer, the application of CNS was attempted with SLNB in a limited sample size of 40 patients in 2017 , with DR of $95 \%$ (38/40), sensitivity of $100 \%$ (5/5), and NPV of $100 \%{ }^{22}$. Therefore, our study was the first prospective study with a large sample size; it demonstrated a promising overall DR (91.29\%), sensitivity (92.86\%), and high NPV (99.29\%), especially for patients with tumours smaller than $20 \mathrm{~mm}(97.75 \%, 100 \%$, and $100 \%$, respectively), thereby indicating its clinical value for SLNB in early-stage cervical cancer.

These indicators mentioned above in the first prospective multi-centre study that enrolled 590 patients, using technetium $\left({ }^{99} \mathrm{mTc}\right)$ and patent blue were $88.6 \%, 77.4 \%$, and $94.3 \%{ }^{23}$. In a recent systematic review, indocyanine green (ICG) used in SLNB had a reported DR of $78 \%$ to $100 \%$ and sensitivity of $50 \%$ to $100 \%^{24}$. However, the tracers reportedly had some limitations. Their dependence on special expensive imaging technology could deter their use in developing countries ${ }^{25}$, additionally, its use is technically and logistically challenging. The ${ }^{99} \mathrm{mTc}$ is expensive and produces radioactive contaminants; The blue dye is associated with allergic reactions, blue discoloration of body fluids, and rare cardiovascular reactions ${ }^{26-27}$. Therefore, compared with the traditional agents mentioned, CNS in SLNB for early-stage cervical cancer not only has good diagnostic accuracy but is also safer, more economical, easier to use, and not dependent on imaging technology.

According to our data, $85.71 \%$ of detected 1456 SLNs were in expected locations (i.e., iliac vessels and obturator). Less common locations were the aortic (6.94\%), presacral $(4.33 \%)$, and parametrial $(3.02 \%)$ regions. This is consistent with the results of a review of 3012 SLNs (blue dye and ${ }^{99} \mathrm{mTc}$ ) ${ }^{28}$. Therefore, SLNB helps surgeons not only during direct SLN resection with less disruption of the lymphatic architecture, thus making the procedure easier and faster ${ }^{18}$, but also in identifying occult SLNs outside the traditional dissection boundaries.

When trying to determine which subgroup of patients can obtain more benefits from SLNB, we found that the DR of patients with $<20 \mathrm{~mm}$ tumours was significantly higher than that of patients with [?]20 $\mathrm{mm}$ tumours. Moreover, the sensitivity, specificity, and NPV of SLNB also improved in patients with $<20 \mathrm{~mm}$ tumours. Similar outcomes were reported in a prospective multi-centre study ${ }^{22}$ and a review that assessed more than 600 cases (blue dye and radio-colloid) ${ }^{29}$. Therefore, SLNB with CNS is a promising procedure for well-selected early-stage cervical cancer patients, such as patients with tumour size $<20 \mathrm{~mm}$.

The FNR in our study is $7.14 \%$ (two false-negative cases with negative pathologic ultrastaging results), and it ranged from $0 \%$ to $50 \%$ in previous studies ${ }^{30}$. We implemented an ideal pathologic ultrastaging method to detect micro-metastasis, to further explore the causes of the two false-negative $\operatorname{cases}^{31}$; negative results were obtained, that have appeared in previous report ${ }^{32}$. Furthermore, the common characteristics of these two patients were summarised: tumour diameters larger than $20 \mathrm{~mm}$ with presence of DSI and LVSI (Table S3). These findings are consistent with those of previous studies that reported that FNR was higher for larger tumours $(>20 \mathrm{~mm})$, those with DSI ([?]50\%), and those with LVSI (blue dye, radio-colloid, and ICG) ${ }^{16,33-34}$. The prevalence can be attributed to the hypothesis that metastatic disease can alter the lymphatic drainage pattern $^{35}$. Cancer cells spreading from the primary cancer mass may block the lymphatic path to the first lymph node or nodes. This may explain why there were few cases with failed SLN detection or false-negative results. Regarding the false-negative results, the lymphatic drainage and tracer may bypass the true SLN, which may be falsely recognised as an alternative node (a true non-SLN) ${ }^{36-37}$. This situation may result in false-negative cases.

Previous studies demonstrated that DSI, LVSI, large tumour size, and positive PLNs were prognostic factors for clinical outcomes ${ }^{38-39}$, and that tumours larger than $20 \mathrm{~mm}$ and positive LVSI and DSI were significant risk factors for pelvic nodal metastasis ${ }^{40-41}$. We also found that tumours size, stage, and LVSI are independent 
risk factors for lymph node metastasis. The results of our study (CNS) are consistent with those of previous studies (blue dye, radio-colloid and ICG), which showed that FNR was higher for larger tumours (>20 mm), those with DSI ([?]50\%) and those with LVSI ${ }^{16,33-354}$. Therefore, to avoid the risk of false-negative cases and improve clinical outcomes, SLNB is more suitable for well-selected early-stage cervical cancer patients, especially in patients with tumours smaller than $20 \mathrm{~mm}$, without DSI and LVSI, which are more likely benefit from SLNB. More clinical studies are required to confirm it.

\section{Conclusion}

High DR, sensitivity, and NPV and low FNR were found in our study. These results suggest the possibility that SLNB with CNS is feasible, safe, and reliable in early-stage cervical cancer. We consider SLNB with CNS performed by surgeons with extensive experience to be an important part of individualised treatment for well-selected early-stage cervical cancer patients.

\section{Acknowledgments}

We would like to thank the participating patients for their help with this study.

\section{Disclosure of Interests}

The authors declare that there are no conflicts of interest.

\section{Contribution to Authorship}

Prof. Guo Ruixia is responsible for the study, who designed and implemented the research. She has done plenty of sentinel lymph node biopsy in early-stage cervical cancer patients followed by standardized treatment for cervical cancer. Dr. Xie Ya, Wang Qian, Wen Haixiao, Zhang Weiwei and Bai Jing have performed sentinel lymph node biopsy followed by standard treatment in early-stage cervical cancer. Yang Jianping and Yan Shuping were in charge of the histopathological evaluation. Li Huiqing, Cai Lei, Mo Jiaya and Wang Dong were responsible for data collection and result analysis. Dr. Xie Ya and Li Huiqing drafted the manuscript. Prof. Guo took part in revising this manuscript. Each author has participated sufficiently in the work to take public responsibility for appropriate portions of the content; and has agreed to be accountable for all aspects of the work in ensuring that questions related to the accuracy or integrity of any part of the work are appropriately investigated and resolved. All authors read and approved the final manuscript.

\section{Details of Ethics Approval}

The study was approved by the Institutional Review Board of the First Affiliated Hospital of Zhengzhou University (2018-KY-41; date of approval 14 August 2018). All patients provided written informed consent.

Funding: sponsored by Program for Science and Technology Innovation Talents in Henan Province (2018JR0004) and ZhongYuan thousand talents program-the Zhongyuan eminent doctor in Henan province (ZYQR201810107) and National Natural Science Foundation of China (31670844).

\section{References}

1 Torre LA, Bray F, Siegel RL, Ferlay J, Lortet-Tieulent J, Jemal A. Global cancer statistics, 2012. CA Cancer J Clin. 2015;65:87-108. DOI:10.3322/caac.21262.

2 Siegel RL, Miller KD, Jemal A. Cancer statistics, 2018. CA Cancer J Clin. 2018;68:7-30. DOI:10.3322/caac.21442.

3 Darai E, Rouzier R, Ballester M, Barranger E, Coutant C. Sentinel lymph node biopsy in gynaecological cancers: the importance of micrometastases in cervical cancer. SURG ONCOL. 2008;17:227-35. DOI:10.1016/j.suronc.2008.04.002.

4 Niikura H, Okamoto S, Otsuki T, Yoshinaga K, Utsunomiya H, Nagase S, et al. Prospective Study of Sentinel Lymph Node Biopsy Without Further Pelvic Lymphadenectomy in Patients 
With Sentinel Lymph Node-Negative Cervical Cancer. INT J GYNECOL CANCER. 2012;22:1244-50. DOI:10.1097/IGC.0b013e318263f06a.

5 Cabanas RM. An approach for the treatment of penile carcinoma. CANCER-AM CANCER SOC. 1977;39:456-66.

6 Tax C, Rovers MM, de Graaf C, Zusterzeel PL, Bekkers RL. The sentinel node procedure in earlystage cervical cancer, taking the next step; a diagnostic review. GYNECOL ONCOL. 2015;139:559-67. DOI:10.1016/j.ygyno.2015.09.076.

7 NCCN Cervical cancer guidelines. Version II.2018; Available from. https://www.nccn.org/professionals/physician_gls/pdf/cervical.pdf; NCCN.org. (2018.11.10).

8 Chambers LM, Vargas R, Michener CM. Sentinel lymph node mapping in endometrial and cervical cancer: a survey of practices and attitudes in gynecologic oncologists. J GYNECOL ONCOL. 2019;30. DOI:10.3802/jgo.2019.30.e35.

9 Abu-Rustum NR, Khoury-Collado F, Gemignani ML. Techniques of sentinel lymph node identification for early-stage cervical and uterine cancer. GYNECOL ONCOL. 2008;111: S44-S50. DOI:https://doi.org/10.1016/j.ygyno.2008.07.027.

10 Di Guilmi J, Darin MC, Toscano M, Maya G. Laparoscopic Sentinel Lymph Node Mapping with Indocyanine Green Using the iSpies Platform: Initial Experience Argentina. J MINIM INVAS GYN. 2018;25:378-9. DOI:https://doi.org/10.1016/j.jmig.2017.09.004.

11 Bricou A, Barranger E, Uzan S, Darai E. Anaphylactic shock during the sentinel lymph node procedure for cervical cancer. GYNECOL ONCOL. 2009;114:375-6. DOI:https://doi.org/10.1016/j.ygyno.2009.04.027.

12 Sun SP, Zhang Y, Cui ZQ, Chen Q, Zhang W, Zhou CX, et al. Clinical application of carbon nanoparticle lymph node tracer in the VI region lymph node dissection of differentiated thyroid cancer. GENET MOL RES. 2014;13:3432-7. DOI:10.4238/2014.April.30.4.

13 Khoury-Collado F, Glaser GE, Zivanovic O, Sonoda Y, Levine DA, Chi DS, et al. Improving sentinel lymph node detection rates in endometrial cancer: How many cases are needed? GYNECOL ONCOL. 2009;115:453-5. DOI:https://doi.org/10.1016/j.ygyno.2009.08.026.

14 Cibula D, Zikan M, Slama J, Fischerova D, Kocian R, Germanova A, et al. Risk of micrometastases in non-sentinel pelvic lymph nodes in cervical cancer. GYNECOL ONCOL. 2016;143:83-6. DOI:10.1016/j.ygyno.2016.07.101.

15 Tanaka T, Sasaki S, Tsuchihashi H, Terai Y, Yamamoto K, Yamada T, et al. Which is better for predicting pelvic lymph node metastases in patients with cervical cancer: Fluorodeoxyglucose-positron emission tomography/computed tomography or a sentinel node biopsy? A retrospective observational study. Medicine (Baltimore). 2018;97:e0410. DOI:10.1097/md.0000000000010410.

16 Dundr P, Cibula D, Nemejcova K, Ticha I, Bartu M, Jaksa R. Pathologic Protocols for Sentinel Lymph Nodes Ultrastaging in Cervical Cancer. Arch Pathol Lab Med. 2019. doi:10.5858/arpa.2019-0249-RA.

17 Yahata H, Kobayashi H, Sonoda K, Kodama K, Yagi H, Yasunaga M, et al. Prognostic outcome and complications of sentinel lymph node navigation surgery for early-stage cervical cancer. INT J CLIN ONCOL. 2018;23:1167-72. DOI:10.1007/s10147-018-1327-y.

18 Lennox GK, Covens A. Can sentinel lymph node biopsy replace pelvic lymphadenectomy for early cervical cancer? GYNECOL ONCOL. 2017;144:16-20. DOI:10.1016/j.ygyno.2016.08.337.

19 Yang SX, Wei WS, Jiang QH, Zhou YF, Qu W, Tu JH, et al. Analysis of 246 sentinel lymph node biopsies of patients with clinical primary breast cancer by application of carbon nanoparticle suspension. J Obstet Gynaecol Res. 2018;44:1150-7. DOI:10.1111/jog.13635. 
20 Li Z, Ao S, Bu Z, Wu A, Wu X, Shan F, et al. Clinical study of harvesting lymph nodes with carbon nanoparticle suspension in advanced gastric cancer: a prospective randomized trial. World J Surg Oncol. 2016;14:88.

21 Zhao WJ, Luo H, Zhou YM, Gou ZH, Wang B, Zhu JQ. Preoperative ultrasound-guided carbon nanoparticle suspension localization for metastatic lymph nodes in papillary thyroid carcinoma during reoperation: a retrospective cohort study. Medicine (Baltimore). 2017;96:e6285.

$22 \mathrm{Lu}$ Y, Wei JY, Yao DS, Pan ZM, Yao Y. Application of carbon nanoparticles in laparoscopic sentinel lymph node detection in patients with early-stage cervical cancer. PLOS ONE. 2017;12:e0183834. DOI:10.1371/journal.pone.0183834.

23 Altgassen C, Hertel H, Brandstaedt A, Koehler C, Duerst M, Schneider A. Multicenter validation study of the sentinel lymph node concept in cervical cancer: AGO study group. J CLIN ONCOL. 2008;26:2943-51. DOI:10.1200/jco.2007.13.8933.

24 Rocha A, Maria Dominguez A, Lecuru F, Bourdel N. Indocyanine green and infrared fluorescence in detection of sentinel lymph nodes in endometrial and cervical cancer staging - a systematic review. Eur J Obstet Gynecol Reprod Biol. 2016;206:213-9. DOI:10.1016/j.ejogrb.2016.09.027

25 Di Guilmi J, Darin MC, Toscano M, Maya G. Laparoscopic Sentinel Lymph Node Mapping with Indocyanine Green Using the iSpies Platform: Initial Experience Argentina. J MINIM INVAS GYN. 2018;25:378-9. DOI:https://doi.org/10.1016/j.jmig.2017.09.004.

26 Barthelmes L, Goyal A, Newcombe RG, McNeill F, Mansel RE. Adverse reactions to patent blue V dye - The NEW START and ALMANAC experience. Eur J Surg Oncol. 2010;36:399-403. DOI:10.1016/j.ejso.2009.10.007.

27 Zakaria S, Hoskin TL, Degnim AC. Safety and technical success of methylene blue dye for lymphatic mapping in breast cancer. AM J SURG. 2008;196:228-33. DOI:10.1016/j.amjsurg.2007.08.060.

28 Ouldamer L, Marret H, Acker O, Barillot I, Body G. Unusual localizations of sentinel lymph nodes in early-stage cervical cancer: A review. Surg Oncol. 2012;21: E153-E7. DOI:10.1016/j.suronc.2012.04.003.

29 Lukas R, Helena R, Jiri HM, Martin H, Petr S. Current status of sentinel lymph node mapping in the management of cervical cancer. Expert Rev Anticancer Ther. 2013;13:861-70. DOI:10.1586/14737140.2013.811147.

30 Darai E, Rouzier R, Ballester M, Barranger E, Coutant C. Sentinel lymph node biopsy in gynaecological cancers: The importance of micrometastases in cervical cancer. Surgical Oncology. 2008;17:227-35. DOI:https://doi.org/10.1016/j.suronc.2008.04.002.

31 Cibula, D., Oonk, M. H. \& Abu-Rustum, N. R. Sentinel lymph node biopsy in the management of gynecologic cancer. "Curr Opin Obstet Gynecol,2.411" 27, 66-72, doi:10.1097/gco.0000000000000133 (2015).

32 Cibula D, Abu-Rustum NR, Dusek L, et al. Bilateral ultrastaging of sentinel lymph node in cervical cancer: lowering the false-negative rate and improving the detection of micrometastasis. Gynecol Oncol. 2012;127(3):462-466.

33 Slama J, Dundr P, Dusek L, Cibula D. High false negative rate of frozen section examination of sentinel lymph nodes in patients with cervical cancer. GYNECOL ONCOL. 2013;129:384-8. DOI:10.1016/j.ygyno.2013.02.001.

34 Kim JH, Kim DY, Suh DS, Kim JH, Kim YM, Kim YT, et al. The efficacy of sentinel lymph node mapping with indocyanine green in cervical cancer. WORLD J SURG ONCOL. 2018;16:52. DOI:10.1186/s12957018-1341-6.

35 O'Boyle JD, Coleman RL, Bernstein SG, Lifshitz S, Muller CY, Miller DS. Intraoperative lymphatic mapping in cervix cancer patients undergoing radical hysterectomy: A pilot study. GYNECOL ONCOL. 
2000;79:238-43. DOI:10.1006/gyno.2000.5930.

36 Moncayo VM, Alazraki AL, Alazraki NP, Aarsvold JN. Sentinel Lymph Node Biopsy Procedures. Semin Nucl Med. 2017;47:595-617. DOI:https://doi.org/10.1053/j.semnuclmed.2017.06.004.

37 Eiriksson, L. R. \& Covens, A. Sentinel lymph node mapping in cervical cancer: the future? "Bjog-An International Journal Of Obstetrics And Gynaecology,5.193" 119, 129-133, doi:10.1111/j.1471-0528.2011.03134.x (2012).

38 Twu NF, Ou YC, Liao CI, Chang WY, Yang LY, Tang YH, et al. Prognostic factors and adjuvant therapy on survival in early-stage cervical adenocarcinoma/adenosquamous carcinoma after primary radical surgery: A Taiwanese Gynecologic Oncology Group (TGOG) study. SURG ONCOL. 2016;25:229-35. DOI:10.1016/j.suronc.2016.05.028.

39 Colturato, L. F., Signorini Filho, R. C., Fernandes, R. C., et al. Lymph node micrometastases in initial stage cervical cancer and tumoral recurrence. Int J Gynaecol Obstet 133, 69-75, doi:10.1016/j.ijgo.2015.08.019 (2016).

40 Chandacham A, Charoenkwan K, Siriaunkgul S. et al. Extent of lymphovascular space invasion and risk of pelvic lymph node metastases in stage IB1 cervical cancer. J Med Assoc Thai 88 Suppl 2, S31-36 (2005).

41 Yanaranop M, Sathapornteera N, Nakrangsee S. Risk factors of pelvic lymph node metastasis in cervical adenocarcinoma following radical hysterectomy and pelvic lymphadenectomy. J Med Assoc Thai. 2014;97 Suppl 11: S87-95.

Table 1. Thepatients' demographic and clinicopathologic informationand analysis of impact factors on the SLNB detection rate

Categorical variables are presented as absolute frequency $(\mathrm{N})$ and relative frequency $(\%)$, continuous variables are presented as mean (min-max), comparison between detection rates indicate significance at $P<0.05$, "a": $\mathrm{P}-$ value of continuous correction chi-square test, SLN: sentinel lymph node, SLNB: sentinel lymph node biopsy, LVSI: lymphovascular space invasion.

Table 2. Diagnostic values of SLNB using carbon nanoparticle suspension

PLN: pelvic lymph nodes, Se: sensitivity, Sp: specificity, PPV: positive predictive value, NPV: negative predictive value, FNR: False negative rate, Ac: accuracy, DR: Detection rate, "positive": positive pathological metastasis result, "negative": negative pathological metastasis result.

Figure 1.The technique ofcarbon nanoparticle suspension application in SLNB

A: Schematic diagram indicated that SLNB was performed by injecting CNS into the vaginal portion of the cervical stroma at the 3 and 9 o'clock positions superficially by a skin test needle, approximately $5-10 \mathrm{~mm}$ from the tumor border and perpendicular to the cervix surface. The needle was inserted approximately $2-3$ $\mathrm{mm}$ into the submucosa, and the suspension was slowly injected in approximately $3 \mathrm{~min}$. B: The black-stained SLN was attached to the external iliac artery and vein, which belonged to SLN of external iliac region.C: Locations of SLNs on each side of the pelvis. D1, D2: Two false negative cases with lymphovascular space invasion (HEx100).

\section{Figure 2.Flowchart of SLNB}

CNS: carbon nanoparticle suspension, SLN mapping (+): SLN black-stained positive, SLN mapping (-): SLN black-stained negative, "+": positive pathological metastasis result, "-": negative pathological metastasis result.

\section{Hosted file}

Tables.docx available at https://authorea.com/users/303406/articles/433548-role-of-carbonnanoparticle-suspension-in-sentinel-lymph-node-biopsy-for-early-stage-cervical-cancer-a- 
prospective-study

\section{Hosted file}

Figure 1..png available at https://authorea.com/users/303406/articles/433548-role-of-carbonnanoparticle-suspension-in-sentinel-lymph-node-biopsy-for-early-stage-cervical-cancer-aprospective-study

\section{Hosted file}

Figure 2. .tif available at https://authorea.com/users/303406/articles/433548-role-of-carbonnanoparticle-suspension-in-sentinel-lymph-node-biopsy-for-early-stage-cervical-cancer-aprospective-study 